\title{
Rivaroxaban reduces serious CV events without increasing fatal bleeding in patients with a recent ACS
}

\begin{abstract}
Results of the ATLAS ACS 2-TIMI 51
trial were released at the 2011 AHA

Scientific Sessions in Orlando, FL, and

published in the New England Journal

of Medicine in November. In patients

with a recent acute coronary syndrome

(ACS), long-term administration of low doses of rivaroxaban (a direct factor Xa inhibitor) was associated with reduced risk of major cardiovascular events with no increase in risk of fatal bleeding. Notably, though, major bleeding and intracranial hemorrhage were increased with rivaroxaban use.
\end{abstract}

ATLAS ACS 2-TIMI 51 was an international, randomized, double-blind, placebo-controlled, event-driven trial of two low doses of rivaroxaban versus placebo in patients (aged $\geq 18$ years) with a recent ACS. Enrollment occurred within 7 days of hospital admission for the index event, and patients aged $<55$ years also required diabetes mellitus or a previous MI to be eligible for inclusion. In addition to rivaroxaban or placebo, all patients received standard medical therapy, such as aspirin and a thienopyridine.

In total, 15,526 patients were randomly assigned to a twice-daily $2.5 \mathrm{mg}$ ('verylow') dose of rivaroxaban, a twice-daily $5 \mathrm{mg}$ ('low') dose of rivaroxaban, or placebo in a 1:1:1 fashion. Of the included patients, 50.3\% had experienced an ST-segment-elevation MI (STEMI), $25.6 \%$ a non-STEMI, and $24.0 \%$ unstable angina to qualify them for the study. Mean time on study treatment was 13.1 months. During the course of treatment, $7.8 \%$ of patients receiving placebo, and $8.7 \%$ and $8.5 \%$ of the patients receiving the very-low and low doses of rivaroxaban, respectively, withdrew their consent for the study.

Rates of the primary composite efficacy end pointdeath from a cardiovascular cause,
MI, or stroke-were lower for the patients treated with the $2.5 \mathrm{mg}$ and $5 \mathrm{mg}$ twicedaily doses of rivaroxaban $(9.1 \%$ and $8.8 \%$, respectively) than for individuals receiving placebo (10.7\%). Hazard ratios for the very-low and low doses of rivaroxaban were 0.84 (95\% CI 0.72-0.97, $P=0.02$ ) and 0.85 (95\% CI 0.73-0.98, $P=0.03$ ), respectively, compared with placebo.

Analysis of the individual components of the primary efficacy end point indicated that the beneficial effects of the very-low dose rivaroxaban were driven by reduction in risk of cardiovascular death. By contrast, the beneficial effects of the twice-daily $5 \mathrm{mg}$ dose of rivaroxaban were not driven by any reduction in risk of cardiovascular death but, rather, by a significant reduction in the risk of MI.

The secondary composite efficacy end point—death from any cause, MI, or stroke-occurred in 9.3\%, 9.1\% and $11.0 \%$ of patients receiving very-low-dose rivaroxaban, low-dose rivaroxaban, and placebo, respectively. Hazard ratios for the very-low and low doses of rivaroxaban were 0.83 (95\% CI 0.72-0.97, $P=0.02$ ) and 0.84 (95\% CI 0.73-0.98, $P=0.02$ ), respectively, compared with placebo.

A reduction in stent thrombosis was also noted for the very-low dose of rivaroxaban $(2.2 \%)$ compared with placebo $(2.9 \%)$; the hazard ratio was 0.65 (95\% CI 0.45-0.94, $P=0.02$ ). A nonsignificant trend for reduced stent

thrombosis was observed in the low-dose rivaroxaban group (2.3\%; hazard ratio $0.73,95 \% \mathrm{CI}$ 0.51-1.04, $P=0.08)$, compared with placebo. On the basis of the stent-thrombosis results, the ATLAS ACS 2TIMI 51 investigators speculate that "enhanced thrombin activity might play a role in [the assessed cardiovascular] events." The rate of the primary safety end point-TIMI major bleeding not related to CABG surgery-was substantially increased in the very-low dose (1.8\%) and low-dose (2.4\%) rivaroxaban groups, compared with in the placebo group (0.6\%). Hazard ratios for the very-low and low doses of rivaroxaban were 3.46 (95\% CI 2.08-5.77, $P<0.001)$ and 4.47 (95\% CI 2.71-7.36, $P<0.001)$, respectively.

Risks of TIMI minor bleeding $(0.9 \%$ and $1.6 \%$ versus $0.5 \%$ ), TIMI bleeding requiring medical attention (12.9\% and $16.2 \%$ versus $7.5 \%$ ), and intracranial hemorrhage ( $0.4 \%$ and $0.7 \%$ versus $0.2 \%)$ were also significantly increased in the patients receiving very-low and low doses of rivaroxaban, respectively, compared with individuals administered placebo. Importantly, however, both very-low-dose and lowdose rivaroxaban were not associated with significantly increased risks of fatal bleeding $(0.1 \%$ and $0.4 \%$, respectively, versus $0.2 \%$ in placebo) or of any other type of adverse event.

Notably, the very-low dose of rivaroxaban was associated with significantly lower rates of TIMI minor bleeding, TIMI bleeding requiring medical attention, and fatal bleeding than the low dose of this oral anticoagulant.

On the basis of their findings, the ATLAS ACS 2-TIMI 51 trial investigators conclude that "when viewed as long-term therapy after an ACS, the addition of verylow-dose rivaroxaban appears to be an attractive option."

Bryony M. Mearns 